\title{
Abstract: Fully Automated Deep Learning Pipeline for Adipose Tissue Segmentation on Abdominal Dixon MRI
}

\author{
Santiago Estrada ${ }^{1}$, Ran $\mathrm{Lu}^{1}$, Sailesh Conjeti ${ }^{1}$, Ximena Orozco $^{1}$, Joana Panos $^{1}$, \\ Monique M.B Breteler ${ }^{1,2}$, Martin Reuter ${ }^{1,3}$ \\ ${ }^{1}$ German Center for Neurodegenerative Diseases (DZNE), Germany \\ ${ }^{2}$ IMBIE, Faculty of Medicine, University of Bonn, Bonn, Germany \\ ${ }^{3}$ Department of Radiology, Harvard Medical School, Boston MA, USA \\ santiago.estrada@dzne.de
}

The accurate quantification of visceral and subcutaneous adipose tissue (VAT and SAT) has become a mayor interest worldwide, given that these tissue types represent an important risk factor of metabolic disorders. Currently, the gold standard for measuring volumes of VAT and SAT is the manual segmentation of abdominal fat images from 3D Dixon magnetic resonance (MR) scans - a very expensive and time-consuming process. To this end, we recently proposed FatSegNet [1] a fully automated pipeline to accurately segment adipose tissue inside a consistent anatomically defined abdominal region. The proposed pipeline is based on our competitive dense fully convolutional network (CDFNet): A new 2D F-CNN architecture that promotes feature selectivity within a network by introducing maximum attention through a maxout activation unit [2]. We show that the proposed network architecture (CDFNet) improves segmentation performance and simultaneously reduces the number of required training parameters. FatSegNet produces highly accurate segmentation results (Dice Scores) on SAT compared to inter-rater variability $(0.975$ vs. 0.982$)$ and outperforms manual raters on the more challenging task of VAT segmentation ( 0.850 vs. 0.788$)$. VAT is a more fine-grained compartment with large shape variation. The pipeline additionally demonstrates very high test-retest reliability (ICC VAT 0.998 and SAT 0.996). Overall, FatSegNet generalizes well to different body shapes, sensitively replicates known VAT and SAT volume effects in the Rhineland Study (a large prospective cohort study), and permits localized analysis of fat compartments.

\section{References}

1. Estrada S, Lu R, Conjeti S, et al. FatSegNet: A Fully Automated Deep Learning Pipeline for Adipose Tissue Segmentation on Abdominal Dixon MRI. Journal of Magnetic Resonance Imaging. 2019;.

2. Goodfellow IJ, Warde-Farley D, Mirza M, et al. Maxout networks. In: Proceedings of the 30th International Conference on International Conference on Machine LearningVolume 28. Atlanta,USA: JMLR. org; 2013. p. III-1319. 